\title{
TTR
}

Traduction, terminologie, re?daction

\section{Susanne de Lotbinière-Harwood. Re-Belle et Infidèle / The Body Bilingual. Toronto-Montréal, Women's Press / Les éditions du remue-ménage, 1991.}

\section{Claude Tatilon}

Volume 5, numéro 1, 1er semestre 1992

La pédagogie de la traduction : questions actuelles (1) et Miscellanées traductologiques

URI : https://id.erudit.org/iderudit/014743ar

DOI : https://doi.org/10.7202/014743ar

Aller au sommaire du numéro

\section{Éditeur(s)}

Association canadienne de traductologie

ISSN

0835-8443 (imprimé)

1708-2188 (numérique)

Découvrir la revue

Citer ce compte rendu

Tatilon, C. (1992). Compte rendu de [Susanne de Lotbinière-Harwood. Re-Belle et Infidèle / The Body Bilingual. Toronto-Montréal, Women's Press / Les éditions du remue-ménage, 1991.] TTR, 5(1), 278-288. https://doi.org/10.7202/014743ar

Tous droits réservés ( $\odot$ TTR: traduction, terminologie, rédaction — Les auteurs, Ce document est protégé par la loi sur le droit d'auteur. L’utilisation des 1992 services d’Érudit (y compris la reproduction) est assujettie à sa politique d'utilisation que vous pouvez consulter en ligne.

https://apropos.erudit.org/fr/usagers/politique-dutilisation/ 


\section{Susanne de Lotbinière-Harwood. Re-Belle et Infidèle/ The Body Bilingual. Toronto-Montréal, Women's Press/ Les éditions du remue-ménage, 1991.}

\section{Les enjeux de la traduction féministe}

L'ouvrage de Susanne de Lotbinière-Harwood intrigue dès l'abord par son titre bilingue et sa construction en miroir - première partie en français, seconde en anglais reprenant à peu près les mêmes thèmes que la première. Très vite, il séduit par l'aisance de son style, la finesse de ses observations. Mais, porteur d'un message non équivoque, il invite surtout à la réflexion: «Comme l'écriture au féminin, dont elle est tributaire, la traduction au féminin se présente comme une activité politique visant à faire apparaître et vivre les femmes dans la langue et dans le monde (premier paragraphe de l'ouvrage, p. 11).» Pour toutes ces raisons, Re-Belle... mérite qu'on s'y arrête longuement.

Pour me diriger dans un ouvrage aussi disert, je ferai usage d'un article de Luise von Flotow, «Feminist Translation: Contexts, Practices and Theories», récemment paru dans TTR (IV-2, 1991, pp. 69-84), qui me servira de lanterne de Diogène. (Je ne cherche pas à insinuer, par cette image antique, que Re-Belle... manque de clarté; on se souviendra, du reste, que c'était en plein jour que le cynique grec promenait sa lanterne dans les rues d'Athènes, à la recherche, ô coïncidence! d'un Homme générique.) Cet article nous fait de la traduction féministe un portrait d'où le flou est exclu: il s'agit d'une démarche traductionnelle qui s'exerce majoritairement du français vers l'anglais sur des œuvres d'écrivaines féministes québécoises et qui trouve son origine dans l'idéologie professée par celles-ci. Leur idéologie, qui est plutôt une contre-idéologie, se caractérise essentiellement par la revendication sociale: elles s'insurgent contre le rapport inégalitaire entre les sexes qui fait des femmes des êtres de deuxième catégorie (le Deuxième Sexe) et réclament l'équité. La traduction féministe, comme l'épithète l'indique, milite pour cette même cause. Partant du constat que le langage (langue et discours) reflète et perpétue l'inégalité sociale et faisant l'hypothèse que son assainissement redresserait les rapports sociaux, les adeptes de ce type de traduction orientent leur action dans deux directions: une direction proprement linguistique, de remise en ordre du langage, et une direction traductologique, de mise en œuvre de stratégies spécifiques. 
La remise en ordre du langage, que l'on juge (non sans raison) assujetti à une «norme patriarcale», passe d'abord par une lutte contre la dépréciation (voire l'oblitération) verbale de la femme - dépréciation inscrite dans les structures linguistiques (gars/garce, par exemple), colportée plus ou moins subtilement par les stéréotypes langagiers («Tu causes, tu causes, c'est tout ce que tu sais faire!») et perpétuée par certaines pratiques conversationnelles imposant une hiérarchie des sexes.

Cette remise en ordre passe aussi par une tentative d'amélioration de la communication verbale, qu'il faut infléchir et rendre plus apte à l'expression du vécu féminin: en créant, au cœur même du langage, ce qu'on pourrait appeler un «sexolecte» féminin, contrepartie de la norme patriarcale.

Au service de textes engagés (littéraires ou non), la traduction féministe fait appel, d'autre part, à différentes stratégies afin de faire mieux passer le message en langue-culture d'arrivée. La recherche inconditionnelle d'efficacité qui gouverne ce type de traduction l'exonère en grande partie de la contrainte traditionnelle de «fidélité» vis-à-vis du texte de départ: le message féministe, jugé essentiel, sera, au besoin, amplifié. D'où, diverses stratégies activistes, malmenant autant la norme écrite que la notion de «traducteur neutre universel», qui ont pour résultat d'imposer le sexolecte féminin, trace ostensible de la «main traduisante».

Voilà ce que j'ai cru lire, pour l'essentiel, dans l'intéressant article de Luise von Flotow. Et que je retrouve sans peine sous la plume de Susanne de Lotbinière-Harwood. Mais reprenons les problèmes un à un.

\section{La condamnation de la norme patriarcale}

$R e-B e l l e . .$. est là-dessus sans équivoque: «La règle de grammaire voulant que le masculin l'emporte sur le féminin n'est pas le résultat du hasard, ni l'expression d'un ordre naturel. Elle reflète plutôt la situation d'infériorité socio-économique, politique, juridique et philosophique des femmes. En français, selon la loi du bon usage, une multitude d'êtres animés de genre féminin doivent s'effacer devant un seul objet inanimé de genre masculin. En ceci le français est non seulement anti-démocratique (la majorité ne l'emporte pas) mais foncièrement injuste. La violence faite aux femmes dans la société est donc inscrite dans le système du genre grammatical [...] Les hommes se comportent dans le monde exactement comme le masculin dans le langage, et les femmes considèrent normale cette règle grammaticale 
qui les fait taire et les rend invisibles. Inversement, le comportement langagier se plie à l'ordre établi: en régime patriarcal, il est dans l'ordre des choses que le masculin l'emporte. Nous sommes inscrites dans et circonscrites par l'il-logisme des règles de grammaire où papa a toujours raison (pp. 14-15).»

Thèse résumée en ces termes, p. 38: «l'économie du féminin dans la langue équivaut à l'occultation des femmes dans la réalité.» Mais pas toujours illustrée de manière convaincante: «Les reportages sur les massacres à l'École polytechnique de Montréal en décembre 1989 ont permis de constater à quel point le système du genre est violent quand, pour désigner quatorze étudiantes assassinées et un meurtrier qui s'est suicidé, on parlait de quinze morts (pp. 34-35).» L'indignation est légitime mais l'explication grammaticale ne tient pas, le commentaire laissant accroire que l'incongruité de la formulation journalistique (qu'il aurait été de bonne guerre de citer in extenso) tient à un phénomène de langue (le genre grammatical du substantif mort) alors qu'il s'agit clairement d'un choix de discours (quinze morts préféré à quatorze victimes).

On touche ici à un vrai débat linguistique: jusqu'où peut-on aller, que peut-on faire pour décorseter la langue française, pour la rendre moins ségrégationniste?

Mais Re-Belle... escamote ce débat. D'abord, en n'appuyant pas ses analyses sur des travaux solides. Marina Yaguello est citée, certes, mais pour un court paragraphe (p. 33) de sens très général; alors qu'une grammairienne néophyte, Françoise Marois, dont les propositions hardies n'ont que la caution de la bonne volonté, est prisée pour sa «nouvelle philosophie grammaticale qui se fonde sur une réalité contemporaine». (Elle propose, entre autres trouvailles, la forme illes pour le personnel sujet 6 , «collectif mixte».) Wendy Martyna est citée à trois reprises (pp. 32, 82, 112) mais seulement pour une expression («he/man language»). L'excessive Julia Penelope l'est aussi, cursivement (p. 152), ainsi que Suzette Haden Elgin (pp. 109, 111), l'auteure de linguistique-fiction, inventrice du láadan («a language constructed by a woman, for women, for the specific purpose of expressing the perceptions of women»). On trouve aussi, çà et là, quelques citations prophétiques, du genre: «il n'y aura plus de féminins en -euse d'ici la fin du siècle. Ils ont acquis une connotation trop péjorative ( $p$. 28, n. 28).» Mais nulle part de références sérieuses, discutées et intégrées à l'ouvrage. Les travaux d'Anne-Marie Houdebine, par exemple, auraient pu être utilisés pour le français, comme ceux de Claire Michard, de 
Catherine Viollet et, bien sûr, de Marina Yaguello; pour l'anglo-américain, ceux de Mary Ritchie Key, de Nancy Henley, Cheris Kramarae, Robin Lakoff, Barrie Thorne.

Débat escamoté aussi, parce que ne sont jamais abordées certaines questions de première importance pour une réflexion sur la féminisation. En premier lieu, celle des automatismes langagiers et de l'arbitraire du signe linguistique, ressort essentiel de la dynamique de la langue, point de départ obligé de toute discussion sur l'expression linguistique du «sexe». La langue est avant tout convention, il est vain de rechercher une correspondance exacte entre les mots et les choses. (J'ai écrit plus haut «sous la plume» de S de L-H: n'aurais-je pas dû? On dit couramment, cinq siècles après Copernic, que «le soleil se lève et se couche»: ne devrait-on pas? L'«atome» n'est-il pas sécable, aujourd'hui? Son «pied» nous force-t-il à voir dans la chaise un objet humanisé? L'expression «prendre ses jambes à son cou» devrait-elle être réservée aux seuls contorsionnistes? Et aux seuls peintres ou chimistes celle de «broyer du noir»?) Il est bon de garder à l'esprit que, le langage fonctionnant à partir d'un faisceau d'habitudes acquises très tôt, une trop grande acuité métalinguistique nuit à son bon fonctionnement.

Nulle part n'est faite, non plus, la distinction, pourtant essentielle, entre le monème «sexe» (qui est un signifié linguistique au sens précis du terme, alors que le gender, notion sociologique chère aux féministes anglophones, n'en est pas un) et le genre grammatical, qui n'est pas un monème et n'a donc pas de signifié (et, qui plus est, n'est plus, en anglais d'aujourd'hui, qu'un rêve de grammairien dans la lune - au fait, comment pourrait-on, littéralement, «être dans la lune»?).

Nulle part, non plus, il n'est dit clairement que la structure linguistique respective de l'anglais et du français oblige à des solutions particulières pour la féminisation dans chacune de ces langues. Ni qu'il est impératif de ne pas confondre, dans quelque langue que ce soit, contraintes de langue et choix de discours.

Je n'ai rien trouvé de tout cela dans $R e-B e l l e . .$. Si bien que la revendication linguistique qui s'en dégage - revendication légitime, certes, et fortement exprimée - ne peut avoir pour effet positif que de sensibiliser son lectorat aux discriminations du langage et de le convaincre de la nécessité de «faire quelque chose». Mais quoi? Il est à craindre qu'une discussion laissant entendre que le problème est simple (qu'il suffit, le plus 
souvent, d'un $\underline{\mathbf{e}}$ bien placé pour venir à bout de la plupart des problèmes posés par la langue française) ait finalement un effet pernicieux et ne fasse qu'encourager la féminisation sauvage - qui, à mon sens, n'a pas besoin de l'être et qui nuit beaucoup à la crédibilité d'un aménagement linguistique et langagier évidemment nécessaire.

\section{La lutte contre la dépréciation verbale de la femme}

Située au plan du discours, l'intervention est ici moins compliquée: il s'agit de mettre en garde - et Re-Belle... est en cela très efficace - contre l'emploi de clichés sexistes («Sois belle, et tais-toi!», «Comme la plume au vent femme est volage...») et d'unités lexicales à teneur négative. Dans ce domaine, où les actes de parole sont très souvent involontaires, il est utile de rendre conscient de la charge malveillante de certains mots ou expressions. «Feminization [...] includes strategies such as avoiding pejorative words designating women, encoding new meanings in existing words and coining new words, after using etymology as a resource.» (p. 117) Ces

recettes sont bonnes à suivre. À condition, cependant, de toujours bien faire le distinguo entre grammaire et lexique, puis entre langue et discours. Le problème est surtout de savoir où s'arrêter. Faut-il suivre $S$ de L-H et condamner à l'oubli belles infideles, expression historiquement datée, parce qu'on y lit en filigrane: «Une traduction, c'est comme une femme: si elle est belle, elle risque d'être infidèle»? (p. 21) Chacun décidera pour soi.

Mais il est temps de reconnaître qu'au niveau du discours les insinuations sexistes sont loin d'être rares. En voici un intéressant exemple, sans doute inconscient, relevé dans le texte de présentation du Petit Robert, édition de 1977: «il s'agit de fournir à tout lecteur les instruments de pensée et d'expression les plus élaborés: l'agriculteur, le travailleur manuel, la ménagère [sic] ou le syndicaliste ont évidemment droit aux mêmes instruments culturels que l'avocat et le médecin.» La présentation de 1978 corrige ainsi le tir: «ll en est de même pour les utilisateurs professionnels, dactylos, secrétaires, rédacteurs et rédactrices, instituteurs et professeurs [...] le grand public».

Sur ce terrain, beaucoup de choses sont encore à faire, et peuvent l'être sans porter atteinte au bon fonctionnement de la langue. Pourquoi refuser d'appeler ambassadrice une femme occupant effectivement un poste d'ambassadeur (nouvelle réalité sociale) sous prétexte que ce substantif est traditionnellement réservé à une épouse d'ambassadeur? Il en est de même pour directrice, ministre (Édith Cresson, premier ministre français?!) ainsi 
que pour un grand nombre d'autres termes désignant des professions, titres ou fonctions. Pourquoi s'entêter à ne pas vouloir adapter le lexique à notre époque, où le statut de la femme connaît de rapides et profonds changements?

Malgré la sévère contrainte que le genre impose au français, il existe dans le discours une matge de manœuvre; il est possible de «ruser avec le masculin grammatical français», comme S de L-H le dit, p. 38, et le démontre avec talent, pp. 67-68. Ayant à traduire un passage de roman dans lequel l'héroïne «raconte un rêve où le référent est birds, "oiseaux" ("The first one was a nightingale [...] The second was attached to the first by a string [...] The third was sitting on a tree [...]")», la traductrice se rend compte que «le sexe de ces oiseaux n'est pas évident à la lecture de l'anglais. Ma première réécriture, explique-t-elle, était donc au masculin. Mais l'auteure m'a précisé qu'il s'agissait d'oiseaux femelles. Vu le niveau de langue parlé du roman (l'héroïne raconte ici son rêve à sa thérapeute), il n'était pas question d'employer "oiselle", mot de niveau plus littéraire. L'auteure et l'éditrice m'ont soumis une version où la féminisation s'effectuait sans être soulignée textuellement. Si je me souviens bien, cela donnait quelque chose comme: "La première, un rossignol (...)" La transition était un peu brusque à mon gout. Mais à l'aide de leurs suggestions, et parce que ce roman utilise souvent le langage pour parler du langage, $j$ 'ai eu recours à cette même approche métalinguistique pour féminiser la narration du rêve: "Le premier - ou plutôt, la première, puisque c'étaient des femelles - un rossignol très modeste, se tenait [...] La deuxième était attachée [...] Elle [...] La troisième était perchée [...]" Le bout de phrase inséré entre les tirets convient bien au niveau parlé du roman et permet de rendre compte d'une stratégie de féminisation très marquée de façon tout à fait naturelle [...] Le décrochage entre les masculins "rossignol", "oiseau" et les féminins "elle", "attachée", "perchée", dérange nos habitudes de lecture, mais il n'est pas plus étrange que le décrochage produit par le bon usage ("madame le ministre" et autres barbarismes).» Bel exemple à suivre.

\section{Fonction expressive et usage poétique du langage}

La mobilisation contre le sexisme verbal concentre donc son action sur la recherche de moyens langagiers pouvant permettre aux femmes de mieux s'exprimer, de mieux dire leur vécu intime, de mieux «transcrire le souffle du corps parlant dans l'écriture» (Re-Belle..., p. 42), d'explorer les expériences féminines «that had not been put into words before» (Luise von 
Flotow, p. 72); et même d'écrire «l'inédit» - «a privileged term in Brossard's writing referring to that which cannot be inscribed for lack of appropriate language». (LvF, p. 72, n. 8)

Il y a là deux choses à ne pas confondre. La première, assez banale, assez facile à réaliser: débâillonner le discours, dire enfin des choses trop longtemps frappées de censure par le «parler-homme" («Our private parts have been thoroughly colonized by male use and abuse», Re-Belle..., p. 148). Parler, par exemple, de la sexualité de la femme, libérer son corps des Blasons qui l'embaument en le fleurissant (à l'exception de celui extraordinaire de tendre érotisme et de lucidité métalinguistique - de Georges Brassens!); la faire revivre verbalement. Au besoin, la décrire crûment.

La seconde, beaucoup moins banale, est bien plus délicate: «we need to find our own words to speak our sexual bodies.» (Re-Belle..., p. 148) Il ne s'agit là de rien de moins que d'écriture poétique.

Parfois, on a le désir, confus mais exigeant, d'exprimer une pensée complexe, une émotion profonde ne pouvant se satisfaire d'une formulation hâtive. La recherche d'une forme adéquate est alors l'occasion d'approfondir sa pensée, d'analyser son émotion. Opération toujours délicate, décevante souvent. Si la réussite n'est pas chaque fois au bout de l'effort, il arrive tout de même que contenu et expression parviennent à faire corps. Les personnes qui se livrent le plus souvent à cette douloureuse maïeutique sont les poètes, plus habiles que les autres à trouver la formule qui dira adéquatement leur pensée, leur émotion, et dans laquelle les lecteurs pourront à leur tour retrouver leur propre vécu. Ce travail d'expression, cette praxis, qui s'exerce sur le matériau linguistique en vue d'en tirer un maximum de sens, on peut commodément l'appeler usage poétique du langage (qu'il ne faut pas confondre avec la fonction poétique, plus étroitement formelle, de Roman Jakobson). Usage que Georges Mounin démonte et décrit depuis de nombreuses années. On lira avec grand profit son demier ouvrage, Sept Poètes et le langage, que Gallimard vient de publier dans la collection Tel. On y constatera que la communication poétique est toujours difficile - pour les écrivains tout autant que pour les écrivaines. On y lira, par exemple, que Ponge se plaint de ce que «les paroles sont toutes faites et s'expriment: elles ne [1]'expriment point»; que Mallarmé poursuit un but sisyphéen: «nier l'indicible»; que Char se livre à «une marche forcée dans l'indicible». De Char encore, cette vision de 
préhistoire: «Il faut réapprendre à frapper le silex à l'aube, s'opposer au flot des mots.»

Peut-on dire que «l'écriture au féminin» enrichit la communication écrite? Sans aucun doute. Que l'on songe aux nombreux écrits littéraires et «manifestaires» (Re-Belle..., p. 62) inspirés par la condition féminine. Enrichissement de la pensée, de toute évidence. Mais aussi enrichissement de la langue, par certaines formes nouvelles comme professeure, écrivaine (Ne disons pas stupidement que «ça rime avec vaine»!), par des changements morphologiques comme médecin, devenu épicène (et entré dans l'usage canadien tout comme les deux noms précédents). Enrichissement du discours poétique tout autant, obtenu de haute lutte par d'acrobatiques et «heureux accidents de langage» que la traduction au féminin n'est pas la dernière à provoquer. Relevés dans Re-Belle...: la segmentation signifiante d'il-logique (S de L-H), la commutation innovatrice d'ellogiquement (idem) ou de scribelle (féminin de scribe), joyau signé Nicole Brossard, des mots-valises du genre de Lovhers, géniale traduction par Barbara Godard d'un titre de roman, Amantes, de Nicole Brossard... Tout cela est brillant, remarquable sur le plan de la création verbale, et capable d'enrichir la langue de nouveaux moyens d'expression. Mais surtout, c'est la nette démonstration que la norme patriarcale n'empêche nullement les femmes de s'exprimer. Les plus belles trouvailles d'écriture proviennent de difficultés vaincues. La thèse n'est pas neuve.

\section{Des stratégies traductionnelles activistes}

À ce propos, deux remarques. La première, qu'il n'y a rien là (dans la «supplémentation», degré ultime de la surtraduction consistant à insérer des commentaires dans le TA pour expliciter la pensée féministe du TD; dans l'utilisation généreuse de «notes» et de «préfaces»; et dans le «détournement» qui consiste à s'approprier le TA et à le mettre au service de ses propres idées - LvF, pp. 74-80) de très original: gloses, surtraductions et usurpations de texte ne datent pas d'hier.

Ma seconde remarque, pour souligner une exagération: le projet féministe (priorité au message féministe du TD, qu'on se permettra de grossir à volonté dans le TA, avec ou sans l'accord de l'auteur traduit) nous est présenté comme «an anti-traditional, aggressive and creative approach to translation», comme une avancée théorique décisive qui devrait renvoyer la notion de "fidélité» au musée de la réflexion traductologique: "The modest, self-effacing translator, who produces a smooth, readable target 
language version of the original has become a thing of the past ( $\mathrm{LvF}, \mathrm{p}$. 76).» Ce qui fait problème ici, et suscite la réprobation, c'est la généralisation abusive à partir d'une perspective au demeurant très particulière. $\mathrm{LvF}$ ajoute: «It seems evident that in devising and practising creative non-traditional approaches to translation, making their presence felt in the texts and challenging their authors, feminist translators in Canada are making changes to some of these traditional views and the habitual "missionary" position assigned to translation. Translation practice [...] can only stand to gain from such new approaches (p. 82).»

S de L-H dit à peu près la même chose: «Contrairement à une pratique de traduction orthodoxe, qui entretient l'illusion de la parfaite neutralité, voire même [sic] de la non-existence de la main traduisante, la traduction comme pratique de réécriture au féminin met cartes sur table dès le départ. Son projet est de faire entrer la conscience féministe dans l'activité traduisante.» (p. 11)

Dans ce passage, l'auteure apparât moins radicale que $L v F$, semblant ne vouloir parler que de «la traduction comme pratique de réécriture au féminin». Mais l'ambiguïté n'est pas complètement dissipée. Citant, p. 26, une jolie formule de portée générale donnant la traduction pour «une ré-écriture dans la langue d'arrivée d'une lecture dans la langue de départ», S de L-H la commente en soulignant l'importance fondamentale de «la présence d'un sujet traduisant», d'une part, et du «lieu de passage, l'espace-entre-deux-langues d'où parle ce sujet traduisant», d'autre part. Mais, ce faisant, elle n'accorde aucune attention à l'information véhiculée par le TD, opportunément occultée dans la citation elle-même. Cela est bien commode: pas de contenu à réexprimer, pas de fidélité à respecter; la main traduisante est libre alors de s'en donner à coeur joie.

L'information textuelle initiale représente bien, pourtant, la raison première de toute traduction. (Arrive-t-il souvent qu'on traduise de mauvais textes seulement parce qu'ils offrent un contenu féministe?) Quoi qu'on dise ou qu'on fasse, la traduction stricto sensu consistera toujours à retrouver d'abord, inscrite dans le texte de départ, l'«expérience humaine» (artistique ou utilitaire, imaginaire ou vécue) qu'y a mise l'auteur; puis à couler cette expérience dans le moule d'une langue autre que celle du texte de départ. Et selon l'ingéniosité du "cerveau» traduisant, cette opération - de «repensée» (André Martinet) - s'effectuera plus ou moins «fidèlement». Si le mot gêne, on pourra toujours lui préférer ceux d'équivalence ou de correspondance. Mais l'idée de reproduction (d'une information textuelle 
diversifiée) continuera à hanter la traductologie, qui ne cessera de s'interroger sur la ressemblance du texte traduit à son modèle et de se prononcer sur la qualité du rendu traductionnel. Le texte de départ existe bel et bien, et son information est en grande partie communicable. Sinon, à quoi bon traduire? La traduction, activité énonciative certes, mais aussi activité dialectique oscillant entre liberté et fidélité, tirant plus d'un côté que de l'autre en fonction de la nature du texte à traduire (souvent un contrat d'assurance, parfois un poème de Mallarmé). $S$ de L-H dit elle-même, excellemment (p. 47): «L'art de traduire réside entre oser et doser.»

Il est clair, en définitive, que la critique du langage est au cour même du féminisme: c'est à un sexolecte tapageur qu'il confie la diffusion de ses idées. Un sexolecte qui est tout à la fois véhicule, symbole et signe de ralliement.

Les manifestations les plus évidentes du sexolecte français sont, d'après LvF: «The silent "e" [...] exploited as a mutant element with which neologisms and puns could be developed to parody and attack conventional language»; puis, plus généralement: «the disregard for grammatical or syntactical structures and the dismantling of individual words in order to examine their concealed meanings (pp. 72-73)»).

Si l'on examine la partie française de Re-Belle..., on constate que $\mathrm{S}$ de L-H fait un usage très fréquent du e pour marquer la double identification sexuelle (graphiquement mise en évidence par des tirets): les colonisé-e-s (p. 16), chacun-e (p. 18), l'employeur-e (p. 30) et qu'elle utilise souvent le doublet: traducteur ou traductrice (p. 23), illelle (p. 28), en accordant parfois la préséance au féminin: la/le guide, celles/ceux, chères/chers (p. 36). Mais, ces procédés ostentatoires, quoique abondants dans l'ensemble du texte, sont généralement utilisés avec circonspection. $S$ de L-H écrit, d'ailleurs: «Quand je parle de la traduction comme d'une pratique de rééćcriture au féminin, il ne s'agit pas, comme le pensent certain-e-s, de tout mettre au féminin (cette curieuse notion que le féminisme n'est qu'un renversement des rôles!). Tout dépend du contexte, je l'ai déjà dit. [Et elle le redira, pp. 53, 57, 68, 72...] Une publicité, un catalogue d'exposition, un rapport annuel, une pièce de théâtre, un roman, offrent des degrés de liberté différents (p. 35).» Il est à souhaiter que cet appel au bon sens et à la modération soit largement entendu.

Trois derniers points pour conclure. 
1. Féminisme anglo, féminisme franco: même combat. Beaucoup de liens les unissent, symbolisés par ces go-between que sont les traducteurs. La lutte contre la norme patriarcale est commune aux deux écoles et emprunte souvent les mêmes chemins. D'où les fréquents amalgames sur le terrain linguistique. On parle volontiers, pour le français comme pour l'anglais, de «masculin» générique. Mais il s'agit, dans un cas, de genre grammatical et, dans l'autre, d'une référence au sexe. Dans le lexique français, à cause précisément du genre grammatical, c'est la dérivation qui prévaut; en anglais, c'est très nettement la composition. Et cela explique en grande partie que, dans la lutte contre l'androcentrisme linguistique, notre langue adopte plus volontiers la stratégie de la féminisation; l'anglais, celle de la neutralisation Dire que ces choses-là sont mal vues constitue un somptueux euphémisme. Il nous manque encore en la matière une solide étude à la Vinay \& Darbelnet.

2. Comme il nous manque un bilan précis des manifestations du sexisme en français - langue et discours. Partant d'une telle problématique, il serait alors possible, sinon aisé, d'entamer des discussions constructives et d'envisager des changements satisfaisants.

3. Les usages idiolectaux très fluctuants des chefs de file du féminisme constituent un encouragement, involontaire mais non moins dangereux, à la féminisation sauvage en français, qui gagne du terrain tous les jours et englue tous les jours un peu plus la communication la plus banale. (Phénomène particulièrement prononcé dans nos universités, par ailleurs légitimement désireuses de se tenir à la pointe de tous les combats pour le bon droit, la justice et l'équité.)

La langue est à tous et à toutes, elle ne peut être modifiée qu'avec l'assentiment de l'ensemble de ses usagers. Le féminisme est un groupe de pression qu'il ne faut pas subir passivement, même (surtout) si l'on en approuve le combat. Il appartient à chacun de nous de créer les conditions d'un vrai débat. En commençant avec nous-même. C'est à quoi incite, me semble-t-il, la lecture de Re-Belle... C'est pourquoi l'ouvrage est à lire d'urgence. $\AA$ « «re-lire» même, in both languages. Mais sans jamais se départir de son esprit critique.

Claude Tatilon Université York 